\title{
Recent results on Kaon decays from NA48/2
}

\author{
A. Bizzeti ${ }^{* \dagger}$ \\ Department of Physics, Informatics and Mathematics, University of Modena and Reggio Emilia \\ and Istituto Nazionale di Fisica Nucleare - Sezione di Firenze, Italy \\ E-mail: andrea.bizzeti@fi.infn.it
}

The first observation of the $K^{ \pm} \rightarrow \pi^{ \pm} \pi^{0} e^{+} e^{-}$rare decay by the NA48/2 experiment at CERN SPS is reported, based on $\sim 2 \times 10^{11} K^{ \pm}$decays recorded in 2003-2004. From a sample of 4919 candidates with $4.9 \%$ background the branching ratio in the full kinematic region is measured to be $\operatorname{BR}\left(K^{ \pm} \rightarrow \pi^{ \pm} \pi^{0} e^{+} e^{-}\right)=(4.24 \pm 0.14) \times 10^{-6}$.

The study of the kinematic space shows evidence for a structure dependent contribution in agreement with predictions based on Chiral Perturbation Theory. The CP-violating asymmetry between $K^{+} \rightarrow \pi^{+} \pi^{0} e^{+} e^{-}$and $K^{-} \rightarrow \pi^{-} \pi^{0} e^{+} e^{-}$decay rates has been measured, providing an upper limit $\left|A_{\mathrm{CP}}\right|<4.82 \times 10^{-2}$ at $90 \% \mathrm{CL}$.

The NA48/2 combined measurement of the charged kaon semileptonic form factors, based on 4.4 million $K^{ \pm} \rightarrow \pi^{0} e^{ \pm} v_{e}$ and 2.3 million $K^{ \pm} \rightarrow \pi^{0} \mu^{ \pm} v_{\mu}$ events collected in 2004 , is presented. This result represents the most precise form factor measurement from a combined $K_{l 3}^{ \pm}$analysis.

XXVII International Workshop on Deep-Inelastic Scattering and Related Subjects - DIS2019

8-12 April, 2019

Torino, Italy

\footnotetext{
* Speaker.

${ }^{\dagger}$ for the NA48/2 Collaboration: G. Anzivino, R. Arcidiacono, W. Baldini, S. Balev, J.R. Batley, M. Behler, S. Bifani, C. Biino, A. Bizzeti, B. Bloch-Devaux, G. Bocquet, N. Cabibbo, M. Calvetti, N. Cartiglia, A. Ceccucci, P. Cenci, C. Cerri, C. Cheshkov, J.B. Chèze, M. Clemencic, G. Collazuol, F. Costantini, A. Cotta Ramusino, D. Coward, D. Cundy, A. Dabrowski, P. Dalpiaz, C. Damiani, M. De Beer, J. Derré, H. Dibon, L. DiLella, N. Doble, K. Eppard, V. Falaleev, R. Fantechi, M. Fidecaro, L. Fiorini, M. Fiorini, T. Fonseca Martin, P.L. Frabetti, L. Gatignon, E. Gersabeck, A. Gianoli, S. Giudici, A. Gonidec, E. Goudzovski, S. Goy Lopez, M. Hita-Hochgesand, M. Holder, P. Hristov, E. Iacopini, E. Imbergamo, M. Jeitler, G. Kalmus, V. Kekelidze, K. Kleinknecht, V. Kozhuharov, W. Kubischta, G. Lamanna, C. Lazzeroni, M. Lenti, L. Litov, D. Madigozhin, A. Maier, I. Mannelli, F. Marchetto, R. Marchevski, G. Marel, M. Markytan, P. Marouelli, M. Martini, L. Masetti, E. Mazzucato, A. Michetti, I. Mikulec, M. Misheva, N. Molokanova, E. Monnier, U. Moosbrugger, C. Morales Morales, D.J. Munday, A. Nappi, G. Neuhofer, A. Norton, M. Patel, M. Pepe, A. Peters, F. Petrucci, M.C. Petrucci, B. Peyaud, M. Piccini, G. Pierazzini, I. Polenkevich, Yu. Potrebenikov, M. Raggi, B. Renk, P. Rubin, G. Ruggiero, M. Savrié, M. Scarpa, M. Shieh, M.W. Slater, M. Sozzi, S. Stoynev, E. Swallow, M. Szleper, M. Valdata-Nappi, B. Vallage, M. Velasco, M. Veltri, S. Venditti, M. Wache, H. Wahl, A. Walker, R. Wanke, L. Widhalm, A. Winhart, R. Winston, M.D. Wood, S.A. Wotton, A. Zinchenko, M. Ziolkowski.
} 


\section{Beam and detectors}

The NA48/2 experiment has been taking data in the years 2003 and 2004, detecting in-flight decays of charged kaons to search for direct $\mathrm{CP}$ violation in $K^{ \pm} \rightarrow 3 \pi$ decays [1]. It used two simultaneous oppositely charged beams of $60 \mathrm{GeV} / \mathrm{c}$ momentum produced by $400 \mathrm{GeV} / \mathrm{c}$ primary CERN SPS protons impinging on a beryllium target. Decays of beam kaons inside a $114 \mathrm{~m}$ long fiducial volume were recorded by downstream detectors.

A magnetic spectrometer, consisting of a dipole magnet and four drift chamber stations, measured trajectories of charged particles with a spatial resolution of $100 \mu \mathrm{m}$, achieving a momentum resolution $\Delta p / p=(1.0 \oplus 0.044 p[\mathrm{GeV} / \mathrm{c}]) \%$. The spectrometer was followed by a scintillator hodoscope (HOD) consisting of two planes segmented into horizontal and vertical strips, with $\sim 150$ ps time resolution.

Photons and electrons were precisely measured by a a Liquid Krypton electromagnetic calorimeter $(\mathrm{LKr})$, consisting of a $27 X_{0}$ almost homogeneous ionization chamber with high-granularity tower read-out, providing an energy resolutions $\Delta E / E=3.2 \% / \sqrt{E[\mathrm{GeV}]} \oplus 9 \% / E[\mathrm{GeV}] \oplus 0.42 \%$ and a position resolution of about $1.5 \mathrm{~mm}$. The ratio $E_{\mathrm{LKr}} / p$ between the energy deposited in the $\mathrm{LKr}$ and the momentum measured by the spectrometer is used for particle identification.

An iron-scintillator hadronic calorimeter (HCAL), three planes of scintillators for muon detection (MUV) and several photon veto detectors completed the experimental apparatus, a detailed description of which can be found in [2].

\section{The $K^{ \pm} \rightarrow \pi^{ \pm} \pi^{0} e^{+} e^{-}$decay}

The $K^{ \pm} \rightarrow \pi^{ \pm} \pi^{0} e^{+} e^{-}$decay, never observed so far, proceeds through virtual photon emission followed by internal conversion into an electron-positron pair, i.e. $K^{ \pm} \rightarrow \pi^{ \pm} \pi^{0} \gamma^{*} \rightarrow \pi^{ \pm} \pi^{0} e^{+} e^{-}$. The virtual photon can be produced by two different mechanisms: Inner Bremsstrahlung (IB) where the virtual photon $\gamma^{*}$ is emitted by the charged mesons in the initial or final state, and Direct Emission (DE) where the $\gamma^{*}$ is radiated off at the weak vertex.

The differential decay rate of this decay consists of three terms: the dominant IB contribution, the DE component (electric $\mathrm{E}$ and magnetic $\mathrm{M}$ parts) and their interference. The IB-M and E-M interference terms are P-odd and cancel upon angular integration in the total rate.

There are few theoretical studies [3, 4, 5] investigating this decay mode. The authors of [4] predicted, using as input the NA48/2 measurements of the $K^{ \pm} \rightarrow \pi^{ \pm} \pi^{0} \gamma$ decay [6], the branching fractions of IB, DE and INT components of the $K^{ \pm} \rightarrow \pi^{ \pm} \pi^{0} e^{+} e^{-}$decay. In a recent revised work [5] they re-evaluated the interference term using more realistic inputs based on additional experimental results and fewer theoretical assumptions.

The $K^{ \pm} \rightarrow \pi^{ \pm} \pi^{0} e^{+} e^{-}$branching fraction is measured relative to the normalization decay $K^{ \pm} \rightarrow \pi^{ \pm} \pi^{0}$, collected concurrently with the same trigger logic. In the signal sample, the $\pi^{0}$ is identified through the dominant $\pi^{0} \rightarrow \gamma \gamma$ mode $\left(\pi_{\gamma \gamma}^{0}\right)$. In the normalization sample, the $\pi^{0}$ is identified through the $\pi^{0} \rightarrow e^{+} e^{-} \gamma$ Dalitz mode $\left(\pi_{D}^{0}\right)$.

Both signal and normalization candidates are selected among events having a three-track vertex with total charge \pm 1 . The photon four-momenta are reconstructed assuming they originate from the three-track vertex. In both (signal and normalization) selections, the mass of the recon- 
structed $\pi^{0}$ and $K^{ \pm}$are required to be within $\pm 15 \mathrm{MeV} / \mathrm{c}^{2}$ and $\pm 45 \mathrm{MeV} / \mathrm{c}^{2}$, respectively, from the corresponding nominal PDG masses [7]. Moreover, the total $\left(\pi^{ \pm} \pi^{0} e^{+} e^{-}\right.$or $\left.\pi^{+} \pi_{D}^{0}\right)$ reconstructed momentum and the position of the 3-track vertex are required to be compatible with the beam momentum and trajectory. The charged pion and the electrons in each $K^{ \pm} \rightarrow \pi^{ \pm} \pi^{0} e^{+} e^{-}$candidate event are identified using kinematics. The single track with charge opposite to $q_{\mathrm{vtx}}$ is assigned the electron mass, then the remaining electron-pion ambiguity for the two same-sign tracks is solved by testing both mass hypotheses $\left(m_{e}, m_{\pi}\right)$ and $\left(m_{\pi}, m_{e}\right)$ and selecting events within a band defined as $\left|\mathrm{M}\left(\pi^{0}\right)-0.42 \mathrm{M}(\mathrm{K})+73.2 \mathrm{MeV} / \mathrm{c}^{2}\right|<6 \mathrm{MeV} / \mathrm{c}^{2}$.

Cuts on the $\pi^{ \pm} \pi^{0}$ and ee $\gamma$ invariant masses are applied to suppress background from $K^{ \pm} \rightarrow$ $\pi^{ \pm} \pi^{0} \pi_{D}^{0}\left(K_{3 \pi D}\right)$ and $K^{ \pm} \rightarrow \pi^{ \pm} \pi_{D}^{0}\left(K_{2 \pi D}\right)$ decays. Signal acceptance has been evaluated from Monte Carlo simulation. More details about this analysis can be found in [8].

Samples of 4919 signal candidates and $16.3 \times 10^{6} K_{2 \pi D}$ candidates have been selected from a subset of $1.7 \times 10^{11}$ Kaon candidates recorded in 2003-2004. Background contamination estimated from Monte Carlo simulatied samples amounts to $(4.9 \pm 0.4) \%$ in the signal mode and to $0.11 \%$ in the normalization mode. The reconstructed $\gamma \gamma$ and $\pi^{ \pm} \pi^{0} e^{+} e^{-}$mass distributions for the selected signal candidates are shown in Fig. 1.

The Branching Ratio of the $K^{ \pm} \rightarrow \pi^{ \pm} \pi^{0} e^{+} e^{-}$decay mode is obtained from the number of signal and normalization events and their estimated background. It results to be $\operatorname{BR}\left(K^{ \pm} \rightarrow\right.$ $\left.\pi^{ \pm} \pi^{0} e^{+} e^{-}\right)=\left(4.24 \pm 0.06_{\text {stat }} \pm 0.03_{\text {syst }} \pm 0.13_{\text {ext }}\right) \times 10^{-6}$, where the statistical error is dominated by the signal statistics, the systematic error by the radiative effects and the external error by the $\pi_{D}^{0}$ branching ratio uncertainty.

This measurement of the $K^{ \pm} \rightarrow \pi^{ \pm} \pi^{0} e^{+} e^{-}$branching ratio is consistent with theoretical predictions [4, 5] $\mathrm{BR}\left(K^{ \pm} \rightarrow \pi^{ \pm} \pi^{0} e^{+} e^{-}\right)=4.183 \times 10^{-6}$ for IB only, $\mathrm{BR}\left(K^{ \pm} \rightarrow \pi^{ \pm} \pi^{0} e^{+} e^{-}\right)=$ $4.229 \times 10^{-6}$ when including all DE and INT terms, although none of the above predictions includes radiative or isospin breaking effects.
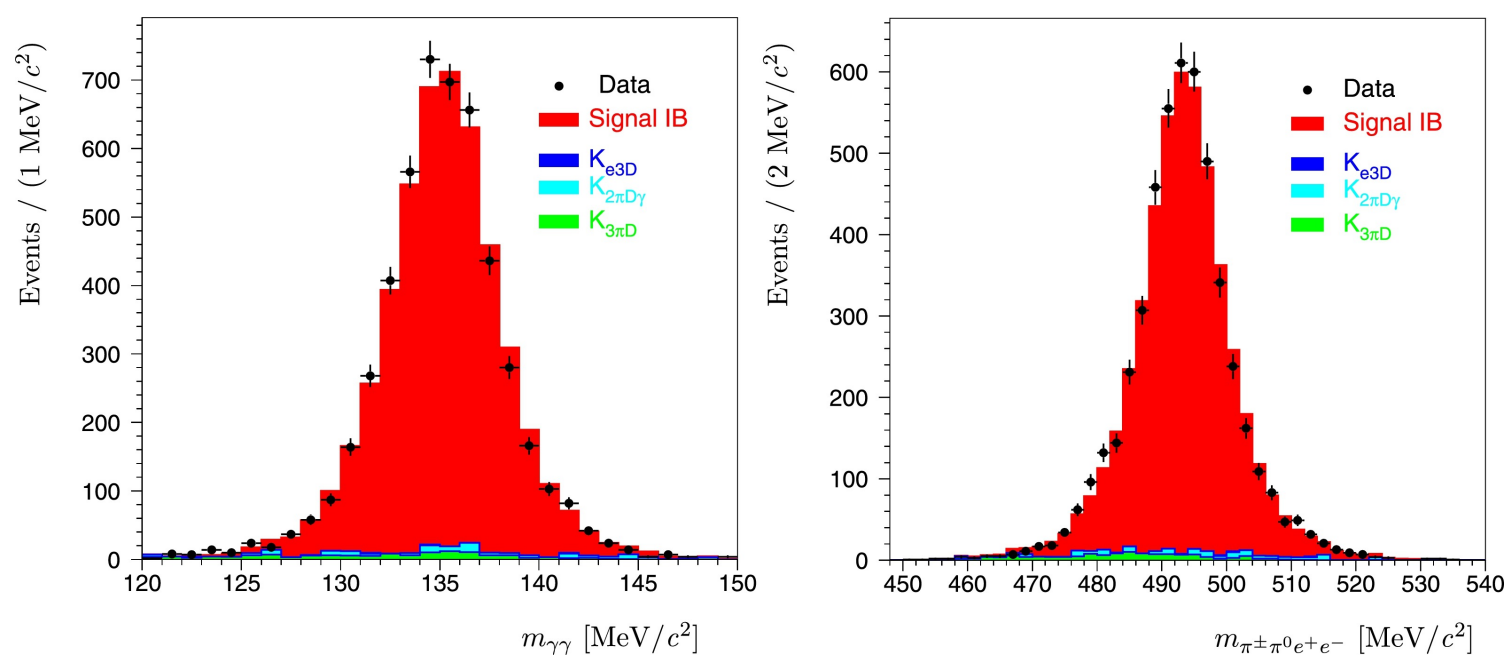

Figure 1: Signal candidates. Left: reconstructed $\gamma \gamma$ mass. Right: reconstructed $\pi^{ \pm} \pi^{0} e^{+} e^{-}$mass. Full dots correspond to data candidates, stacked histograms are, from bottom to top, the expected backgrounds and IB signal (red) estimated from simulation.

A study of the event distribution in the kinematic space allows to determine the ratio between 
$\mathrm{DE}(\mathrm{M})$ and IB contributions to the decay, $M / I B=0.0114 \pm 0.0043_{\text {stat }}$, consistent with the predicted value from [4], $0.0141 \pm 0.0014_{\text {ext }}$. The measurement of both $K^{+}$and $K^{-}$decay rates allows to constrain the CP-violating asymmetry $A_{\mathrm{CP}}=\left[\Gamma\left(K^{+} \rightarrow \pi^{+} \pi^{0} e^{+} e^{-}\right)-\Gamma\left(K^{-} \rightarrow \pi^{-} \pi^{0} e^{+} e^{-}\right)\right] /\left[\Gamma\left(K^{+} \rightarrow\right.\right.$ $\left.\left.\pi^{+} \pi^{0} e^{+} e^{-}\right)+\Gamma\left(K^{-} \rightarrow \pi^{-} \pi^{0} e^{+} e^{-}\right)\right]$, resulting in an upper limit $\left|A_{\mathrm{CP}}\right|<4.82 \times 10^{-2}$ at $90 \% \mathrm{CL}$. More details about these analyses can be found in [8], together with measurements of other P-and $\mathrm{CP}$ - violating asymmetries involving the event distribution in the kinematic space.

\section{Measurement of the $K^{ \pm} \rightarrow \pi^{0} e^{ \pm} v\left(K_{e 3}\right)$ and $K^{ \pm} \rightarrow \pi^{0} \mu^{ \pm} v\left(K_{\mu 3}\right)$ form factors}

The $K_{l 3}(l=e, \mu)$ data sample was collected during a dedicated 4-day period in 2004, with a trigger selecting events with one charged track in the spectrometer and a minimum energy of $10 \mathrm{GeV}$ deposited in the LKr. Offline event selection requires at least two isolated energy clusters in the $\mathrm{LKr}$ consistent with photons of energy above $3 \mathrm{GeV}$, and the sum of their energies above $15 \mathrm{GeV}$. The decay vertex longitudinal position $Z_{\mathrm{v}}$ is reconstructed from the photon energies and positions at $\mathrm{LKr}$, assuming they are produced in the decay of a $\pi^{0}$, i.e. constraining their invariant mass $M_{\gamma \gamma}$ to be consistent with the $\pi^{0}$ mass value [7].

A charged track is also required, with a momentum above $5 \mathrm{GeV} / \mathrm{c}(10 \mathrm{GeV} / \mathrm{c})$ for the $K_{e 3}$ $\left(K_{\mu 3}\right)$ selection. Tracks with $E_{\mathrm{LKr}} / p>0.9$ are identified as electrons or positrons, while muon identification is based on the MUV. The decay vertex transverse position $\left(X_{\mathrm{v}}, Y_{\mathrm{v}}\right)$ is defined by back-extrapolating the track at $z=Z_{\mathrm{v}}$. A wide $Z_{\mathrm{v}}$-dependent cut is applied to the distance between the vertex and the beam axis to include most events produced in the decay of a $3 \%$ additional beam halo component. The kaon momentum $P_{K}$ is determined assuming the kaon line of flight along the beam axis and a massless missing neutrino. From the two possible $P_{K}$ solutions $\left(P_{1}\right.$ and $\left.P_{2}\right)$, the one closest to the beam momentum central value is chosen.

Specific kinematic cuts [9] are applied to the $K_{e 3}$ selection to reduce dependence on the beam shape details and to $K_{\mu 3}$ selection to suppress $K_{2 \pi}$ and $K_{3 \pi}$ backgrounds.

Finally, for both selections the vertex position is required to be consistent (within experimental uncertainty) with a point on the beam axis. The Dalitz plots distributions of the selected samples, consisting of 4.4 million $K_{e 3}$ and 2.3 million $K_{\mu 3}$ events, are shown in Figure 2.

Background contamination is estimated from Monte Carlo simulated samples of several $K^{ \pm}$ decay modes and results very small, $<0.1 \%$ in $K_{e 3}, \sim 0.2 \%$ in $K_{\mu 3}$.

Three different parametrizations are used to describe the $K_{l 3}^{ \pm}$form factors dependence on the $l v$ invariant mass squared: a Taylor expansion [7], a parametrization assuming vector and scalar pole masses $M_{V}$ and $M_{S}[10,11]$ and a more physical dispersive parametrization [12]. The corresponding parameters are determined by minimising a proper $\chi^{2}$ estimator [9]. The fit is performed separately for the $K_{e 3}$ and $K_{\mu 3}$ samples or jointly by extending the summation over both Dalitz plot with a common set of fit parameters. The resulting form factors parameters $\lambda_{+}^{\prime \prime}, \lambda_{+}^{\prime}, \lambda_{0}^{\prime}$ of the Taylor expansion parametrization [7] and their correlations are shown in Figure 3 for the joint $K_{l 3}^{ \pm}$analysis and compared to measurements by previous experiments. The NA48/2 result represents the most precise form factor measurement from a combined $K_{l 3}^{ \pm}$analysis. More details about this analysis, as well as the complete set of results, are reported in [9]. 

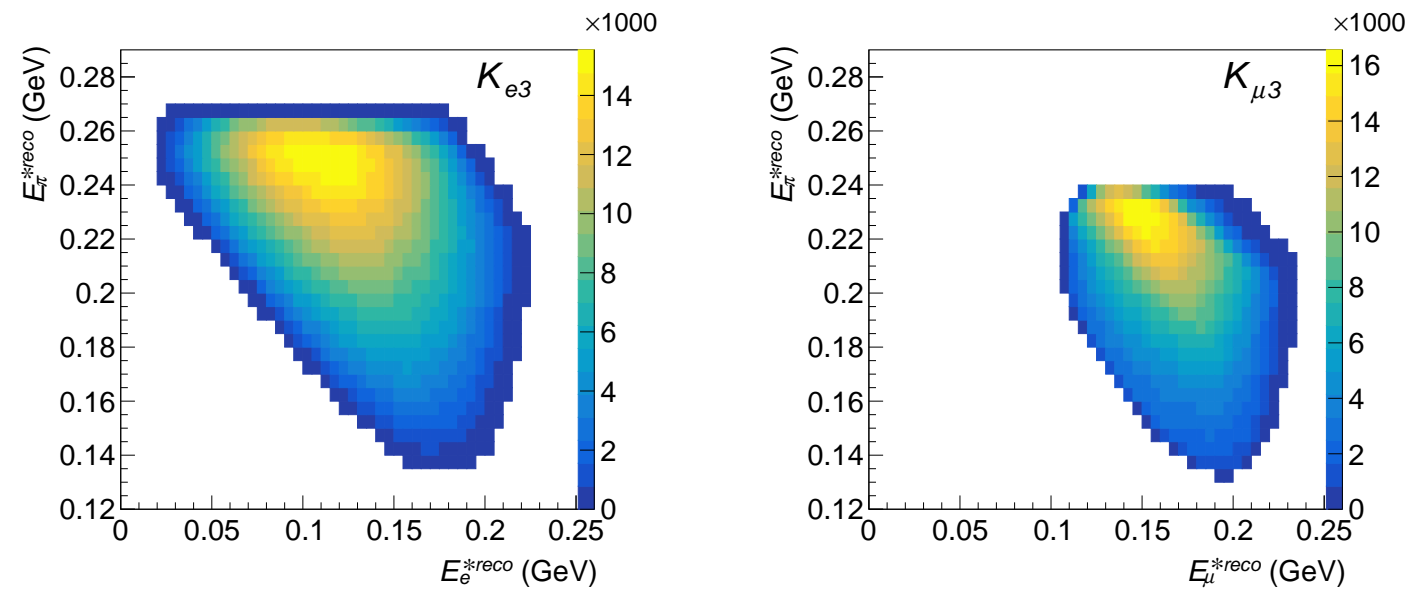

Figure 2: Dalitz plot distribution (in $5 \mathrm{MeV} \times 5 \mathrm{MeV}$ cells) of $K_{e 3}$ (left) and $K_{\mu 3}$ (right) selected events. $E_{\pi}^{* \text { reco }}$ and $E_{l}^{* \text { reco }}(l=e, \mu)$ are the pion and charged lepton energies in the Kaon rest frame.
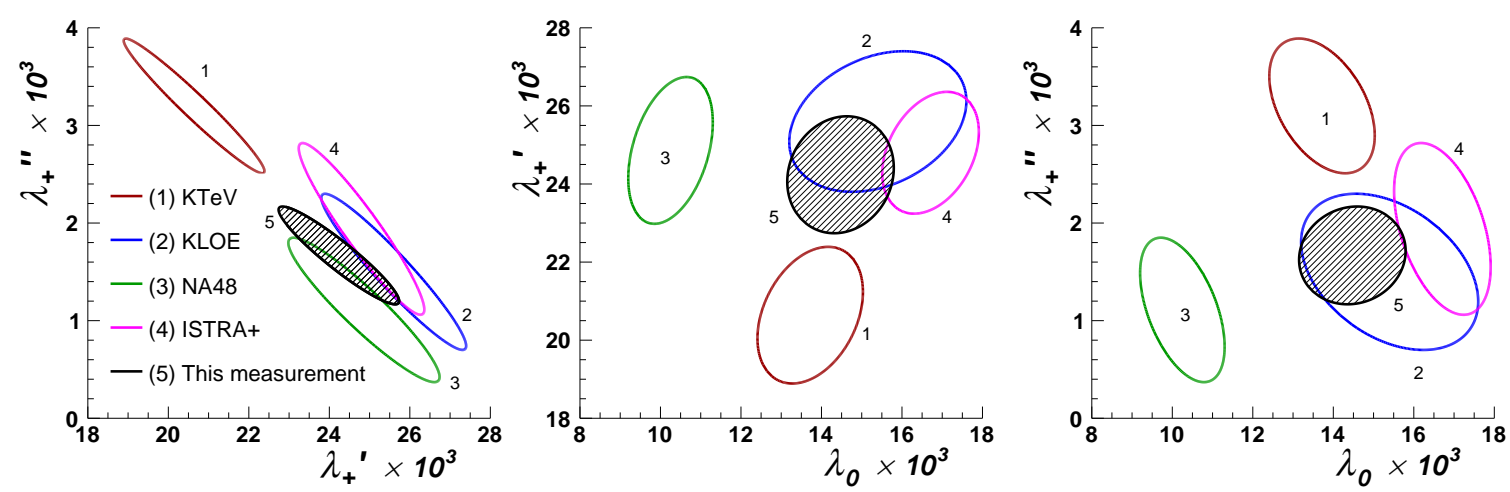

Figure 3: Correlation plots ( $1 \sigma$ contours) of $K_{l 3}^{ \pm}$form factors parameters (Quadratic parametrization) from $K_{e 3}+K_{\mu 3}$ joint analysis. NA48/2 results are shown, together with those of previous experiments.

\section{References}

[1] J.R. Batley et al. [NA48/2 Collaboration], Eur. Phys. J. C 52 (2007) 875.

[2] V. Fanti et al. [NA48 Collaboration], Nucl. Instr. Methods A 574 (2007) 433.

[3] H. Pichl, Eur. Phys. J. C 20 (2001) 371.

[4] L. Cappiello, O. Cata, G. D’Ambrosio and D.N. Gao, Eur. Phys. J. C 72 (2012) 1872.

[5] L. Cappiello, O. Cata, G. D’Ambrosio Eur. Phys. J. C 78 (2018) 265.

[6] J.R. Batley et al. [NA48/2 Collaboration], Eur. Phys. J. C 86 (2010) 75.

[7] M. Tanabushi et al. [Particle Data Group], Phys. Rev. D 98 (2018) 030001.

[8] J.R. Batley et al. [NA48/2 Collaboration], Phys. Lett. B 788 (2019) 552.

[9] J.R. Batley et al. [NA48/2 Collaboration], JHEP 10 (2018) 150.

[10] P. Dennery and H. Primakoff, Phys. Rev. 131 (1963) 1334.

[11] P. Lichard, Phys. Rev. D 55 (1997) 5385.

[12] V. Bernard, M. Oertel, E. Passemar, J. Stern, Phys. Rev. D 80 (2009) 034034. 